\title{
MUTUAL INTERFERENCE AND STRUCTURAL PROPERTIES OF OBJECT IMAGES IN THE VICINITY OF THE GRAVITATIONAL LENS CUSP POINT
}

\author{
A.V. MANDZHOS \\ Astronomical Observatory of Kiev University \\ Observatorna St., 3 Kiev 254053 Ukraine \\ e-mail:aoku@gluk.apc.org
}

Gravitational lens system could form multiple images of the same radiating surface of a cosmic object. The radiation fluxes of these images interfere to one another (Refsdal, 1964). The practical importance of this effect is seems to be as follows: (i) it is the only direct test of microlensing (Schneider et al. 1985); (ii) this effect may say about fine structure of a lensed object; (iii) it must be allowed for simulations of microlensing process. The maximum effect is expected in the case when a cusp point of the microlensing caustic is projected on the lensed object. To investigate the interference effect in this case, the exact solution of the lens equation in the vicinity of the cusp point was obtained (Mandzhos,1993):

$$
\begin{aligned}
& \mathrm{x}_{1,2}=2|\mathrm{~A}| \cdot \cos \left\{\Phi_{0}+[1 \pm \operatorname{sign}(\xi)] \frac{\pi}{3}\right\} \cdot \operatorname{sign}(\xi), \\
& \mathrm{x}_{3}=2|\mathrm{~A}| \cdot \cos \left\{\Phi_{0}+\frac{4}{3} \pi\right\} \cdot \operatorname{sign}(\xi), \quad \mathrm{y}=\frac{1}{2} \eta-\frac{1}{4} \mathrm{vx}^{2}
\end{aligned}
$$

were $\xi, \eta$ are dimensionless coordinates of a radiating element on the object plane; $\mathrm{x}, \mathrm{y}$ are coordinates of the point at which the lens plane is intersected by light beam; furthermore:

$$
\begin{aligned}
& \Phi_{0}=\frac{1}{3} \operatorname{Arctan} \sqrt{\mathrm{h} \frac{\eta^{3}}{\xi^{2}}-1},|\mathrm{~A}|=\mathrm{k} \eta^{1 / 2} \\
& \mathrm{~h}=\frac{2}{9} \frac{\mathrm{v}^{3}}{3 \mathrm{v}^{2}-2 \mathrm{u}}, \quad \mathrm{k}=\sqrt{\frac{2 \mathrm{v}}{3 \mathrm{v}^{2}-2 \mathrm{u}}} .
\end{aligned}
$$

where $u=\partial^{4} \psi / \partial \mathrm{x}^{4} ; \quad \mathrm{v}=\partial^{3} \psi / \partial \mathrm{x}^{2} \partial \mathrm{y}, \psi$ is the lens scalar potential.

M. Kafatos and Y. Kondo (eds.), Examining the Big Bang and Diffuse Background Radiations, 533-534.

(C) 1996 IAU. Printed in the Netherlands. 
It is well known, that there are three images of the internal cusp point region of the object. The mutual coherence degree $\gamma_{i j}$ of the ij-th image pair is the main theoretical quantity in this problem; its module can vary from 0 to 1 . The mutual coherence degree was calculated analytically for a homogeneous disk with the center at the cusp point (Mandzhos, 1993):

$$
\gamma_{13}=\mathrm{P} \cdot\left(\frac{\mathrm{r}_{g}}{c}\left(1+\mathrm{z}_{d}\right)\right)^{-1 / 3} \cdot \mathrm{R}^{-2 / 3}(1-\mathrm{hR})^{5 / 2} \omega^{-1 / 3}
$$

where $\mathrm{P}, \mathrm{h}$ are the dimensionless lens parameters of order unity, $\mathrm{r}_{g}$ is the gravitational radius of the microlens, $\mathrm{z}_{d}$ is the redshift of the lens, $\mathrm{R}$ is the dimensionless radius of the object and $\omega$ is the circular frequency of radiation. The result for the optical range is presented in Fig. 1. Analyzing the real situation in astrophysics, it may be inferred that the actual manifestation of the mutual

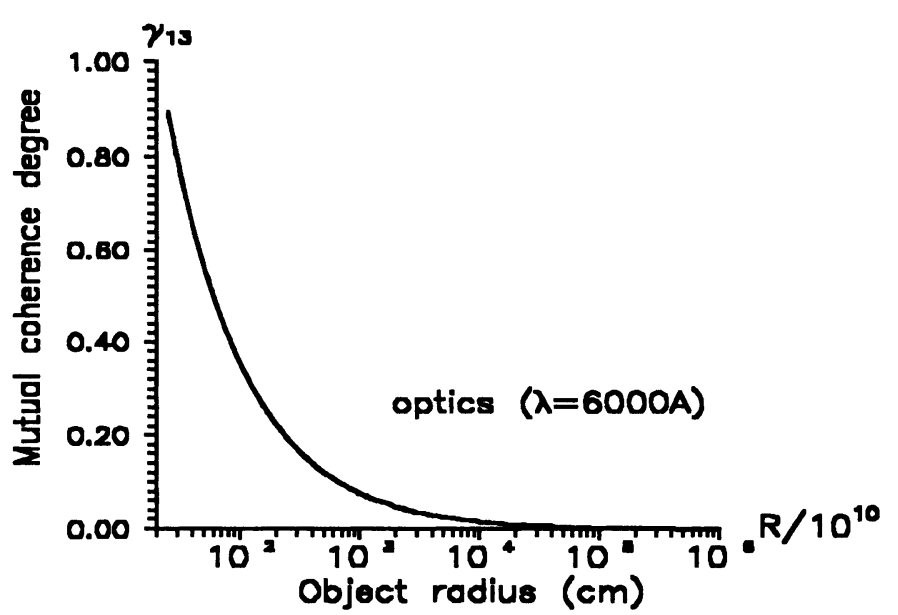
interference effect takes place the following cases: (i) microlensing of a quasar core in radio region; (ii) microlensing of emission clouds from BLR of a quasar in optics; (iii) microlensing of a star from the Magellan Clouds by both stars or MACHO.

Fig. 1. The mutual coherence degree of the radiation from the 1 th and the $3 r d$ microlens images of the internal cusp region of the object. The lens geometry corresponds to the Einstein Cross (Q2237+030)

\section{References}

Mandzhos A.V. 1993 Mutual interference and structural properties of object images in the vicinity of the gravitational lens cusp point. SAO RAS prepr. No.98 Refsdal S. 1964 Mon. Not. Roy. Astron. Soc. 128295

Schneider P. and Schmid-Burgk J. 1985 Astron. Astrophys. 2369 